\title{
The Local Asymptotic Stability of a Water Resource Competition Model with Diffusion of Poisonous Weeds and Allee Effect
}

\author{
Hua Liu ${ }^{1}$, Lei Shi ${ }^{1}$, Yumei Wei ${ }^{2, *}$, Ming Ma ${ }^{1}$ and Jianhua $\mathrm{Ye}^{1}$ \\ ${ }^{1}$ School of Mathematics and Computer Science, Northwest Minzu University, Lanzhou, 730030, Gansu, China \\ ${ }^{2}$ Experimental Center, Northwest Minzu University, Lanzhou, 730030, Gansu, China \\ ${ }^{*}$ Corresponding author
}

\begin{abstract}
Sufficient conditions are obtained for the local asymptotic stability of a water resource competition model with diffusion of poisonous weeds and Allee effect. It is shown that diffusion and Allee effect can increase the possibility of species renewal.
\end{abstract}

\section{Keywords—stability; competition; diffusion; Allee effect}

\section{INTRODUCTION}

Recently, in [1], Hua Liu investigated the persistence of a water resources competition model with Allee effect and diffusion, and the diffusion term is $\mu\left(y_{j}-y_{i}\right)$.

Above analysis motivated us to propose the following two plant species water resource competitive system with diffusion of poisonous weeds and Allee effect:

$$
\left\{\begin{array}{l}
\frac{d x_{i, j}}{d t}=r_{1} x_{i, j}\left(\left(1-\frac{x_{i, j}}{K_{1}}\right)\left(1-\frac{A+c}{x_{i, j}+c}\right)-\alpha \frac{\lambda_{2} V}{r_{1} V_{1}} y_{i, j}\right) \\
\frac{d y_{i, j}}{d t}=r_{2} y_{i, j}\left(1-\frac{y_{i, j}}{K_{2}}-\beta \frac{\lambda_{1}}{r_{2}} \frac{V}{V_{2}} x_{i, j}\right)+\mu\left(\sum y_{i \pm 1, j \pm 1}-4 y_{i, j}\right)
\end{array}\right.
$$

where $x_{i, j}, y_{i, j}$ show the population density of edible forage and poisonous weeds of $(i, j)$ patches; the parameter $K_{i}(i=1,2)$ is the carrying capacity[2]; $r_{i}>0(i=1,2)$ is the intrinsic rate of growth; the parameter $\alpha, \beta>0$ respectively indicates the coefficient of proportionality of moisture loss in root soil layer caused by edible forage and poisonous weeds consumes water resources; $0<\lambda_{i}<1(i=1,2)$ is respectively express utilization of water resources for edible forage and poisonous weeds, and $\lambda_{1}+\lambda_{2}=1 ; V_{i}(i=1,2)$ is the water resource warehouse of edible grass and poisonous weeds, $V=V_{1} \cap V_{2}$ is the overlapping parts of the two water resource warehouse( they co competes resource in $V$ )[1].

In this article, the Allee effect is $\left(1-\frac{A+c}{x_{i, j}+c}\right)$, among $\mathrm{A}$ is the Allee threshold ( $A>0$ is the strong Allee effect , $A<0$ is the feeble Allee effect), $C$ is the auxiliary parameter $(c>0, c \geq-A)$. When $A+c=0$, the Allee effect expression value is 1 indicated that the forage grass had no Allee effect. The parameter $C$ affects the growth curve of edible grass, the curve will flatten when it increase[3-5].

The Cellular Automata will design by Von Neumann type, and each cell has four neighbors. The diffusion term $\mu\left(\sum y_{i \pm 1, j \pm 1}-4 y_{i, j}\right)$ in this system reflects the invasion and diffusion of $(i, j)$ patches of poisonous weeds' neighbor to the $(i, j)$ patches. $\mu$ is the diffusion coefficient[6,7].

\section{MAIN RESUlT}

First, Assume $R_{+}^{2}=\left\{x_{i, j} \geq 0, y_{i, j} \geq 0\right\}$, abandon the negative equilibrium points of ecological significance. The model (1) has four equilibrium points, respectively as follows

$$
\begin{gathered}
p_{1}\left(0, \theta_{1}\right) ; p_{2}\left(0, \theta_{2}\right) ; p_{3}\left(\frac{K_{1}\left(H-\delta_{1} \theta_{3}{ }^{\prime}\right)}{H}, \theta_{3}{ }^{\prime}\right) ; \\
p_{4}\left(\frac{K_{1}\left(H-\delta_{1} \theta_{4}{ }^{\prime}\right)}{H}, \theta_{4}{ }^{\prime}\right) ;
\end{gathered}
$$

where

$$
\begin{gathered}
\delta_{1}=\alpha \frac{\lambda_{2}}{r_{1}} \frac{V}{V_{1}}, \quad \delta_{2}=\beta \frac{\lambda_{1}}{r_{2}} \frac{V}{V_{2}}, H=1-\frac{A+c}{x_{i, j}+c}, \\
\theta_{1}=\frac{1+\sqrt{1+\frac{4 I}{r_{2}} \frac{1}{K_{2}}}}{\frac{2}{K_{2}}}, \quad \theta_{2}=\frac{1-\sqrt{1+\frac{4 I}{r_{2}} \frac{1}{K_{2}}}}{\frac{2}{K_{2}}}, \\
\theta_{3}{ }^{\prime}=\frac{\left(\delta_{2} K_{1}-1\right)+\sqrt{\left(\delta_{2} K_{1}-1\right)^{2}-\frac{4}{r_{2}} \frac{1}{K_{2} H}\left(\delta_{1} \delta_{2} K_{1} K_{2}-H\right) I}}{\frac{2\left(\delta_{1} \delta_{2} K_{1} K_{2}-H\right)}{K_{2} H}},
\end{gathered}
$$




$$
\theta_{4}{ }^{\prime}=\frac{\left(\delta_{2} K_{1}-1\right)-\sqrt{\left(\delta_{2} K_{1}-1\right)^{2}-\frac{4}{r_{2}} \frac{1}{K_{2} H}\left(\delta_{1} \delta_{2} K_{1} K_{2}-H\right) I}}{\frac{2\left(\delta_{1} \delta_{2} K_{1} K_{2}-H\right)}{K_{2} H}},
$$

let parameter $I$ represents the $\mu\left(\sum y_{i \pm 1, j \pm 1}-4 y_{i, j}\right)$ item. (1),

Theorem 1 For homologous self-control system of model

The equilibrium point $p_{1}\left(0, \theta_{1}\right)$ is locally asymptotically stable when $1-\delta_{1} \theta_{1}<0, \theta_{1}>\frac{K_{2}}{2}$;

The equilibrium point $p_{2}\left(0, \theta_{2}\right)$ is locally asymptotically stable when $1-\delta_{1} \theta_{2}<0, \theta_{2}>\frac{K_{2}}{2}$;

The equilibrium point $p_{3}\left(\frac{K_{1}\left(H-\delta_{1} \theta_{3}{ }^{\prime}\right)}{H}, \theta_{3}{ }^{\prime}\right)$ is locally asymptotically stable when $H-\delta_{1} \theta_{3}{ }^{\prime}<0$,

$$
\theta_{3}{ }^{\prime}>\max \left\{\frac{K_{2}}{2}, \frac{K_{2}\left(\delta_{2} K_{1}-H\right)}{2\left(\delta_{1} \delta_{2} K_{1} K_{2}-H\right)}\right\} ;
$$

The equilibrium point $p_{4}\left(\frac{K_{1}\left(H-\delta_{1} \theta_{4}{ }^{\prime}\right)}{H}, \theta_{4}{ }^{\prime}\right)$ is locally asymptotically stable when $H-\delta_{1} \theta_{4}{ }^{\prime}<0$,

$$
\theta_{4}{ }^{\prime}>\max \left\{\frac{K_{2}}{2}, \frac{K_{2}\left(\delta_{2} K_{1}-H\right)}{2\left(\delta_{1} \delta_{2} K_{1} K_{2}-H\right)}\right\} .
$$

Proof: In the following proofs for simplified symbols, $x_{i, j}$ record $X, y_{i, j}$ record $Y$.

According to the qualitative theory of ordinary differential equation,

$$
\begin{gathered}
f(X, Y)=r_{1} H X-\frac{r_{1} H}{K_{1}} X^{2}-r_{1} \delta_{1} X Y, \\
g(X, Y)=r_{2} Y-\frac{r_{2}}{K_{2}} Y^{2}-r_{2} \delta_{2} X Y+I, \\
f_{X}=r_{1} H-\frac{2 r_{1} H}{K_{1}} X-r_{1} \delta_{1} Y, \quad f_{Y}=-r_{1} \delta_{1} X, \\
g_{X}=-r_{2} \delta_{2} Y, \quad g_{Y}=r_{2}-2 \frac{r_{2}}{K_{2}} Y-r_{2} \delta_{2} X .
\end{gathered}
$$

The Jacobian matrix of the system (1) is given by

$$
A=\left[\begin{array}{ll}
f_{X} & f_{Y} \\
g_{X} & g_{Y}
\end{array}\right]=\left[\begin{array}{cc}
r_{1} H-\frac{2 r_{1} H}{K_{1}} X-r_{1} \delta_{1} Y & -r_{1} \delta_{1} X \\
-r_{2} \delta_{2} Y & r_{2}-\frac{2 r_{2}}{K_{2}} Y-r_{2} \delta_{2} X
\end{array}\right] .
$$

Let the characteristic equation is

$$
\lambda^{2}+p \lambda+q=0 .
$$

1) the characteristic equation coefficient of the equilibrium point $p_{1}\left(0, \theta_{1}\right)$ is

$$
\begin{gathered}
p=-\left.\left(f_{X}+g_{Y}\right)\right|_{\left(0, \theta_{1}\right)}=-\left(r_{1}\left(1-\delta_{1} \theta_{1}\right)+r_{2}\left(1-2 \frac{\theta_{1}}{K_{2}}\right)\right), \\
q=\operatorname{det} A=r_{1} r_{2}\left(1-\delta_{1} \theta_{1}\right)\left(1-2 \frac{\theta_{1}}{K_{2}}\right) .
\end{gathered}
$$

One has equilibrium point $p_{1}\left(0, \theta_{1}\right)$ is locally asymptotically stable when $p>0, q>0$ [8-11], then

$$
1-\delta_{1} \theta_{1}<0,1-2 \frac{\theta_{1}}{K_{2}}<0 \text {. }
$$

So, the equilibrium point $p_{1}\left(0, \theta_{1}\right)$ is locally asymptotically stable when $1-\delta_{1} \theta_{1}<0, \theta_{1}>\frac{K_{2}}{2}$.

2) Similarly, the equilibrium point $p_{2}\left(0, \theta_{2}\right)$ is locally asymptotically stable when $1-\delta_{1} \theta_{2}<0, \theta_{2}>\frac{K_{2}}{2}$.

3) the characteristic equation coefficient of the equilibrium point $p_{3}\left(\frac{K_{1}\left(H-\delta \theta_{1} \theta^{\prime}\right)}{H}, \theta_{3}{ }^{\prime}\right)$ is

$$
\begin{gathered}
p=\left(r_{1}+\frac{r_{2} \delta_{2} K_{1}}{H}\right)\left(H-\delta_{1} \theta_{3}{ }^{\prime}\right)+r_{2}\left(1-2 \frac{\theta_{3}{ }^{\prime}}{K_{2}}\right), \\
q=r_{1} r_{2}\left(\delta_{1} \theta_{3}^{\prime}-H\right)\left(1-2 \frac{\theta_{3}^{\prime}}{K_{2}}\right)+r_{1} r_{2} \delta_{2} \frac{K_{1}\left(\delta_{1} \theta_{3}^{\prime}-H\right)}{H}\left(2 \delta_{1} \theta_{3}^{\prime}-H\right) .
\end{gathered}
$$

we obtain $\theta_{3}{ }^{\prime}>\frac{K_{2}}{2}, H-\delta_{1} \theta_{3}{ }^{\prime}>0$ by $q>0$ [8-11].

Again, applying $H-\delta_{1} \theta_{3}{ }^{\prime}>0, q>0$ we get

$$
K_{2} H-2 \theta_{3}{ }^{\prime} H-\delta_{2} K_{1} K_{2} H+2 \delta_{1} \delta_{2} K_{1} K_{2} \theta_{3}{ }^{\prime}>0,
$$

that is

$$
\theta_{3}{ }^{\prime}>\frac{K_{2}\left(\delta_{2} K_{1}-H\right)}{2\left(\delta_{1} \delta_{2} K_{1} K_{2}-H\right)} .
$$


In summary, the equilibrium point $p_{3}\left(\frac{K_{1}\left(H-\delta_{1} \theta_{3}{ }^{\prime}\right)}{H}, \theta_{3}{ }^{\prime}\right)$ is locally asymptotically

stable when $H-\delta_{1} \theta_{3}{ }^{\prime}<0, \theta_{3}{ }^{\prime}>\max \left\{\frac{K_{2}}{2}, \frac{K_{2}\left(\delta_{2} K_{1}-H\right)}{2\left(\delta_{1} \delta_{2} K_{1} K_{2}-H\right)}\right\}$.

4) Similarly available, the equilibrium point $p_{4}\left(\frac{K_{1}\left(H-\delta_{1} \theta_{4}{ }^{\prime}\right)}{H}, \theta_{4}{ }^{\prime}\right)$ is locally asymptotically stable when $H-\delta_{1} \theta_{4}{ }^{\prime}<0, \theta_{4}{ }^{\prime}>\max \left\{\frac{K_{2}}{2}, \frac{K_{2}\left(\delta_{2} K_{1}-H\right)}{2\left(\delta_{1} \delta_{2} K_{1} K_{2}-H\right)}\right\}$.

\section{NUMERICAL EXAMPLE}

Discretize the model, the population dynamics with and without Allee effect was simulated with time $t$ as the horizontal axis, the population of edible herbage is the vertical axis. Let $r_{1}=0.4, r_{2}=0.8, \mu=0.2, A=0.3, c=0.2, K_{1}=1.2$, $K_{2}=1.5, \alpha=0.4, \beta=0.8, \lambda_{1}=\sin ^{2} t, \lambda_{2}=\cos ^{2} t$, $\frac{V}{V_{1}}=\frac{V}{V_{2}}=0.2, x(0)=0.6, y(0)=0.7$.

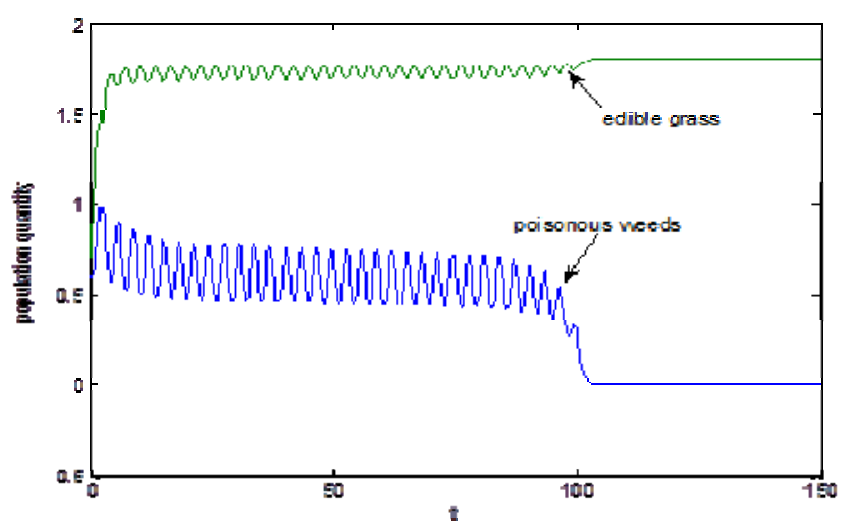

FIGURE I. THE NUMBER OF POPULATION CHANGES WITH TIME WITH ALLEE EFFECT

Figure 1 shown that edible grasses is increasing and poisonous weeds species populations is decreasing at beginning; but when time $t$ is 100 , edible grass species to extinction and poisonous weeds has reached the carrying capacity.

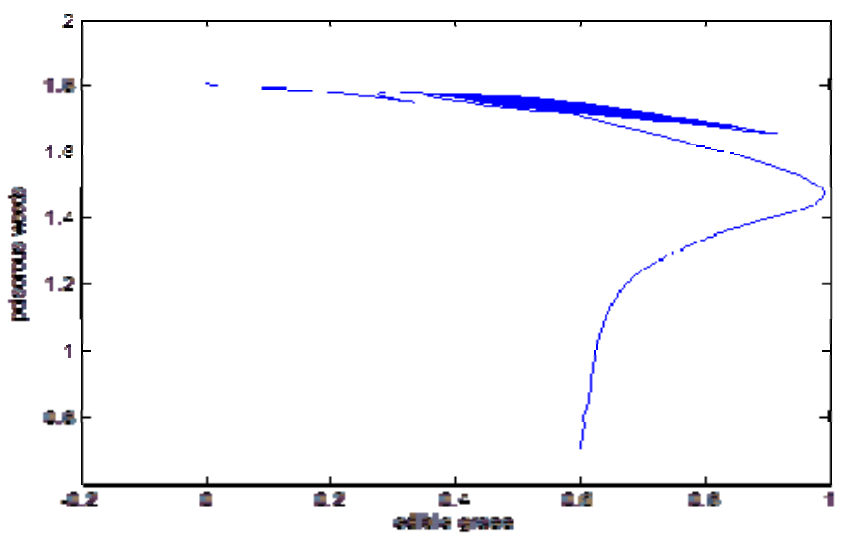

FIGURE II. WITH EDIBLE GRASS POPULATIONS FOR THE HORIZONTAL AXIS, WITH POISONOUS WEEDS POPULATIONS FOR THE LONGITUDINAL AXIS, THE SIMULATION OF THE PHASE DIAGRAM RAIL LINE WITH ALLEE EFFECT

From figure 2, it can be seen that the number of population of edible herbage population and poisonous weed population increased at the same time. When the population of edible herbage population was 0.9 and the population of poisonous weeds was 1.7 , the population of edible herbage began to decrease gradually, while the number of poisonous weeds continued to increase. When the population of edible herbage was 0.5 , the population of the poisonous weeds was 1.65 , and the two populations gradually showed a small change in cycle. Then the population of edible herbage continued to decrease, the population of poisonous weeds continued to increase, and finally the population of herbage was extinct, and the population of poisonous weeds reached their own carrying capacity.

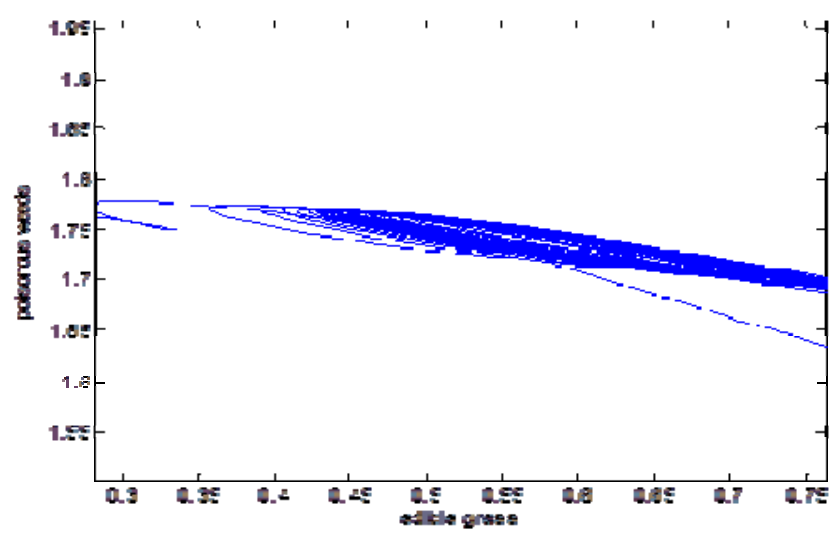

FIGURE III. THE LOCAL ENLARGED IMAGE OF FIGURE 2 


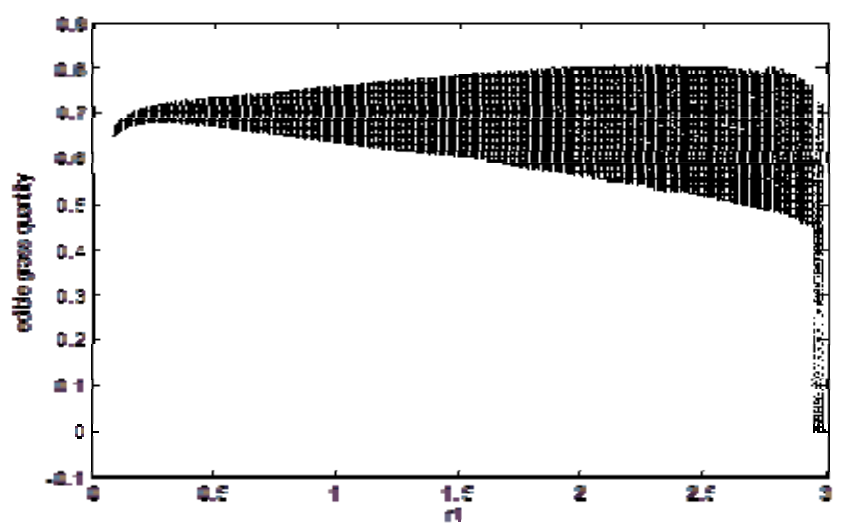

FIGURE IV. WITH EDIBLE GRASSES OF THE INTRINSIC GROWTH RATE FOR THE HORIZONTAL AXIS, WITH EDIBLE FORAGE POPULATION DENSITY AS A NUMBER LINE, THE BIFURCATION DIAGRAM WITH ALLEE EFFECT

Figure 4 shown that the edible grass intrinsic rate of population of the cycle is presented before 2.7 dynamic, after edible herbage species becomes to extinction with invasion of poisonous weeds and Allee effect.

The above numerical simulation also fully verifies the theoretical derivation.

\section{CONCLUSION}

The results shown that there are two asymptotic stability situations in the population of poisonous weeds and edible herbage, and the competition of water resources and the factors of invasion and Allee effect can increase the adaptation of poisonous weeds.

\section{ACKNOWLEDGMENT}

This work is supported by the Fundamental Research Funds for the Central Universities (31920180116, 31920180044), the National Natural Science Foundation of China (31260098, 11361049, 31560127), the Program for Yong Talent of State Ethnic Affairs Commission of China (No.[2014]121), the 2018 laboratory opening project of Northwest Minzu University (SYSKF-2018225, SYSKF-2018236) and the Gansu Provincial First-Class Discipline Program of Northwest Minzu University.

\section{REFERENCES}

[1] Lei Shi, Hua Liu, Yumei Wei, et al. The persistence of invasion and diffusion model of poisonous weeds with Allee effect[J]. International Conference on Applied Mathematics, Modeling and Simulation. 2017, 153:159-163.

[2] Xu R, Chaplain MAJ, Davidson FA. Persistence and global stability of a ratio-dependent predator-prey model with stage structure[J]. Applied Mathematics and Computation. 2004, 158: 729-744.

[3] Zu J. Global qualitative analysis of a predator-prey system with Allee effect on the prey species[J]. Mathematics and Computers in Simulation. 2013, 94: 33-54.

[4] Jang RJ. On the Lotka-Volterra competition system with Allee effects[J]. Computational and Applied Mathematics. 2013, 32: 179-189.

[5] L A, S E, E K, al. e. Competition models with Allee effects[J]. Journal of Difference Equations and Applications. 2014, 20: 1127-1145.

[6] Takeuchi Y. Diffusion-mediated persistence in two-species competition Lotka-Volterra model[J]. Mathematical Biosciences. 1989, 95(1): 65-83.
[7] Zhang XA, Chen L. The linear and nonlinear diffusion of the competitive Lotka-Volterra model[J]. Nonlinear Analysis Theory Methods and Applications. 2007, 66: 2767-2776.

[8] Chicone C. Stability Theory of Ordinary Differential Equations[M]. Computer Algebra Systems A Practical Guide. 2013.

[9] Sideris TC. Ordinary Differential Equations and Dynamical Systems[J]. Atlantis Studies in Differential Equations. 2013, 140: 189-194.

[10] Rado A. Stability of dynamical systems[J]. Differential Equations. 2004 40: 1096-1105.

[11] Lasalle JP, Lasalle JP. Stability theory for ordinary differential equations[J]. Journal of Differential Equations. 1968, 4: 57-65. 